\title{
A stochastic filter for fluid motion tracking
}

\author{
Anne Cuzol \\ Etienne Mémin
}

\author{
IRISA, Université de Rennes 1, \\ Campus de Beaulieu, \\ 35042 Rennes Cedex, France. \\ $\{$ acuzol,memin\}@irisa.fr
}

\begin{abstract}
In this paper we present a method for the tracking of fluid flows velocity fields. The technique we propose is formalized within sequential Bayesian filter framework. The filter we propose here combines an Itô diffusion process coming from a stochastic formulation of the vorticity-velocity form of Navier-Stokes equation and discrete measurements extracted from an image sequence. The resulting tracker provides robust and consistent estimations of instantaneous motion fields along the whole image sequence. In order to handle a state space of reasonable dimension for the $s$ tochastic filtering problem, we represent the motion field as a combination of adapted basis functions. The used basis functions ensue from a mollification of Biot-Savart integral and a discretization of the vorticity and divergence map$s$ of the fluid vector field. The efficiency of the method is demonstrated on a long real world sequence showing a vortex launch at tip of airplane wing.
\end{abstract}

\section{Introduction}

In a number of domains the analysis of image sequences involving fluid phenomenon is of the highest importance. Let us for instance cite the domain of geophysical sciences such as meteorology and oceanography where one wants to track cloud systems for weather forecasting or for surveillance purpose, estimate ocean streams or monitor the drift of passive entities such as icebergs or pollutant sheets. The analysis of fluid flow images is also crucial in experimental fluid mechanics to analyze flows around wing tips or vortex shedding from airfoils or cylinders.

For all the kinds of aforementioned applications and domains it is of major interest to track along time the most accurately as possible representative structures of the flow. Such a tracking which remains to estimate Lagrangian drifters for the structures of interest may be obtained from deterministic integration methods such as the Euler method or the Runge and Kutta integration technique. These numerical integration approaches rely on a continuous spatio- temporal vector field description and thus requires the use of interpolation schemes over the whole spatial and temporal domain of interest. As a consequence, they are quite sensitive to local errors measurements or to inaccurate motion estimates. When the images are noisy or if the flow velocities are of high magnitude and chaotic as in the case of turbulent flows for instance, motion estimation tends to be quite difficult and prone to errors. Another source of error is inherent to motion estimation techniques (see for instance [2] for an extended review on motion estimation techniques). As a matter of fact, most of the motion estimation approaches use only a small set of images (usually two consecutive images of a sequence) and thus may suffer from a temporal unconsistency from frame to frame. The extension of spatial regularizers to spatio-temporal regularizers [16] or the introduction of simple dynamical constraint in motion segmentation techniques relies mainly on crude dynamic assumptions or that are related only to rigid object motion [10].

In order to improve the tracking of dynamical structures from fluid flows image sequence, we propose a tracking method allowing a robust and temporal consistent estimation of instantaneous motion fields along the whole image sequence. In order to be robust to acquisition noise or to illumination conditions the proposed technique is formalized within the Bayesian recursive filter framework.

To settle such a filter, we are facing three main difficulties. The first one concerns the dimension of the state space associated to a fluid motion velocity field. As a matter of fact, Monte-Carlo probabilistic tracking methods [9, 11, 14] are mainly built to track objects of reduced dimension such as points or curves described by several discrete control points. These techniques are not able to cope with high dimensional features such as dense vector fields. In order to handle motion fields of manageable dimension, we describe first an original parametrization of fluid flows relying on adequate basis functions. The used basis functions stem from Biot-Savart integration of a regularized discretization of the vector field vorticity and divergence maps. Such a 
representation enables a parcimonius representation of a fluid motion. The second difficulty is related to the continuous nature of the involved dynamic evolution law. The problem consists thus in the definition of an appropriate sequential Monte-Carlo approximation of a stochastic filter which combines a continuous dynamical law expressed as a stochastic differential equation and discrete measurements extracted from the image sequence. The third difficulty lies in the definition of the dynamic law itself. This law must be sufficiently simple to be efficiently implemented and sufficiently precise to predict the most accurately as possible the evolving motion field.

This paper is organized as follows. After recalling some notions about vector fields, we describe first the low dimensional representation of fluid motion on which we rely. In a second part of the paper we describe the Bayesian filter we devised for fluid motion tracking.

\section{Parametric fluid motion estimator}

\subsection{Some notions about $2 \mathrm{D}$ vector fields}

A two-dimensional vector field $\mathbf{w}$ is a $\mathbb{R}^{2}$-valued map defined on a bounded set $\Omega$ of $\mathbb{R}^{2}$. We denote it $\mathbf{w}(\mathbf{x})=$ $(u(\mathbf{x}), v(\mathbf{x}))^{T}$, where $\mathbf{x}=(x, y)$ and $x$ and $y$ denote the spatial coordinates. Each component of the vector field will be supposed twice continuously differentiable: $u, v \in$ $C^{2}(\Omega, \mathbb{R})$.

Noting $\boldsymbol{\nabla}=\left(\frac{\partial}{\partial x}, \frac{\partial}{\partial y}\right)$ the operator whose components are the partial derivatives with respect to the coordinates $x$ and $y$, we define the divergence: $\operatorname{div} \mathbf{w}=\frac{\partial u}{\partial x}+\frac{\partial v}{\partial y}=$ $\boldsymbol{\nabla} . \mathbf{w}$ and the scalar vorticity of the vector field: $\operatorname{curl} \mathbf{w}=$ $\frac{\partial u}{\partial y}-\frac{\partial v}{\partial x}=\boldsymbol{\nabla} \cdot \mathbf{w}^{\perp}$, where $\mathbf{w}^{\perp}=(-v, u)$ is the orthogonal counterpart of $\mathbf{w}$.

The vorticity accounts for the presence of a rotating motion, while the divergence is related to the presence of sinks or sources in the flow. A vector field whose divergence is null at every point is called solenoidal. Similarly, a field with zero vorticity will be called irrotational. It is well known that for irrotational fields there exists a scalar function $\phi$, called the velocity potential, such that $\boldsymbol{w}=\boldsymbol{\nabla} \phi$. Similarly, for solenoidal fields there exists a scalar function $\psi$ called the stream function such that $\boldsymbol{w}^{\perp}=\nabla \psi$.

Any continuous vector field that vanishes at infinity can be decomposed into a sum of an irrotational componen$\mathrm{t}$ with null vorticity and a solenoidal component with null divergence. This is called the Helmholtz Decomposition. When the null border condition can not be imposed, an additional component, named the laminar component, which is both irrotational and solenoidal, has to be included. The decomposition reads then: $\mathbf{w}=\mathbf{w}_{\text {irr }}+\mathbf{w}_{\text {sol }}+\mathbf{w}_{\text {lam }}$. This last component can be approximated using the Horn and Schunck estimator with a strong regularization coefficient
[6]. In the sequel we will assume that the laminar component has been previously computed and that its associated motion has been removed from the image sequence. We will consequently assume a null boarder condition at infinity knowing that the image sequence, $I(\mathbf{x}, t)$, is related to the original image sequence, $I_{o}(\mathbf{x}, t)$, by $I(\mathbf{x}, t)=$ $I_{o}\left(\mathbf{x}+\mathbf{w}_{\text {lam }}(\mathbf{x}, t), t\right)$.

Substituting the two components $\mathbf{w}_{i r r}$ and $\mathbf{w}_{\text {sol }}$ by their expressions in terms of potential functions and considering the divergence and the curl of the motion field enables to write the potential function as solution of two Poisson equations:

$$
\Delta \phi=\operatorname{divw} \text { and } \Delta \psi=- \text { curlw },
$$

where $\Delta$ denotes the Laplacian operator. These solutions may be expressed as convolution products:

$$
\phi=G * \operatorname{div} \mathbf{w} \quad \text { and } \quad \psi=-G * \operatorname{curl} \mathbf{w},
$$

where $G$ is the Green's function associated to the twodimensional Laplacian: $G(\mathbf{x})=\frac{1}{2 \pi} \ln (|\mathbf{x}|)$.

As the vector fields $\mathbf{w}_{i r r}$ and $\mathbf{w}_{\text {sol }}$ are respectively the gradient and the orthogonal gradient of the potential functions $\phi$ and $\psi$, equations (2) may be rewritten as:

$$
\mathbf{w}_{i r r}=K * \operatorname{div} \mathbf{w} \quad \text { and } \quad \mathbf{w}_{\text {sol }}=-K^{\perp} * \operatorname{curl} \mathbf{w},
$$

where $K$ denotes the gradient of the Green kernel. The second equation of (3) is known as the Biot-Savart integral. These two equations state that the solenoidal and the irrotational components (and consequently the whole vector field) may be recovered through a convolution produc$\mathrm{t}$ knowing the divergence and the vorticity of the velocity field.

\subsection{Vortex and source particles}

The idea of vortex particles methods $[4,12]$ consists in representing the vorticity distribution of a field by a set of discrete amounts of vorticity (vortices). Using these vortices, the vorticity distribution is approximated as a weighted discrete sum:

$$
\operatorname{curl} \mathbf{w}(\mathbf{x}) \approx \sum_{i} \gamma_{i} \delta\left(\mathbf{x}-\mathbf{x}_{i}\right)
$$

where $\mathbf{x}_{i}$ is the location of the vortex $i, \gamma_{i}$ the strength of the vortex and $\delta$ the Dirac delta function. The vortices are called point vortices since they are represented by delta functions.

This discretization of the vorticity into a limited number of elements enables to evaluate the velocity field directly from the Biot-Savart integral (equ. 3). However, because of the singularity of the Green kernel gradient $K$, the velocity field becomes unbounded if any two vortices come 
very close to each other. In fact, the induced field develop$\mathrm{s} \frac{1}{r}$-type singularities, where $r$ is the distance to the point vortices.

These singularities can be removed by smoothing the Dirac measure with a cutt-off or blob function, leading to a smoothed version of $K$. Let $f_{\epsilon}$ be such a blob function scaled by a parameter $\epsilon$ : $f_{\epsilon}(\mathbf{x})=\frac{1}{\epsilon^{2}} f\left(\frac{\mathbf{x}}{\epsilon}\right)$. The smoothed kernel is defined as $K_{\epsilon}=K * f_{\epsilon}$. The amount of smoothing is determined by the value of $\epsilon$. If $\epsilon \rightarrow 0, f_{\epsilon}$ tends to the Dirac function and $K_{\epsilon} \rightarrow K$.

As for the divergence map a similar source particles representation reads then:

$$
\operatorname{div} \mathbf{w}(\mathbf{x}) \approx \sum_{i=0}^{n} \gamma_{i} f_{\epsilon_{i}}\left(\mathbf{x}-\mathbf{x}_{i}\right),
$$

where $\mathbf{x}_{i}$ denotes the center of each basis function $f_{\epsilon_{i}}$, the coefficient $\gamma_{i}$ is the strength associated to the particle $i$, and $\epsilon_{i}$ represents its influence domain. These parameters are free to vary from a function to another.

\subsection{Estimation from consecutive images}

As we saw previously, discretizing the vorticity map with vortex particles together with a smoothing of the Dirac measure leads through Biot-Savart integral to the following representation of the solenoidal component of the motion field:

$$
\mathbf{w}_{\text {sol }}(\mathbf{x}) \approx \sum_{i=0}^{p} \gamma_{i}^{s o l} K_{\epsilon_{i}^{s o l}}^{\perp}\left(\mathbf{x}_{i}^{s o l}-\mathbf{x}\right),
$$

where $K_{\epsilon_{i}}^{\perp}$ is a new kernel function obtained by convolving the orthogonal gradient of the Green kernel with the blob function. Obviously, a similar representation of the irrotational component can be obtained using source particles.

As a result, we exhibit an approximation of the complete motion field as weighted sums of basis functions defined by their center location and respective spatial influence. With a Gaussian smoothing function which allows to derive analytically the associated smoothed kernel $K_{\epsilon}$, the final expression of the solenoidal component is:

$\mathbf{w}_{\text {sol }}(\mathbf{x}) \approx \sum_{i=0}^{p} \gamma_{i}^{s o l} \frac{\left(\mathbf{x}_{i}^{s o l}-\mathbf{x}\right)^{\perp}}{2 \pi\left|\mathbf{x}-\mathbf{x}_{i}^{s o l}\right|^{2}}\left(1-\exp \left(-\frac{\left|\mathbf{x}-\mathbf{x}_{i}^{s o l}\right|^{2}}{\epsilon_{i}^{s o l^{2}}}\right)\right)$.

The irrotational component is expressed through source particles and a similar orthogonal expression.

This representation is incorporated within a spatiotemporal variation model of the luminance function in order to devise fluid motion recovery as an estimation problem from the image sequence data.

For image sequences showing evolving fluid phenomena, the usual brightness consistency assumption $\left(\frac{d I}{d t}=0\right)$ doesn't allow to model temporal distortions of luminance patterns caused by 3D matter transportation. For such kind of sequences, several works have shown that a data model build from an analogy with the mass conservation constraint of fluid mechanics (also known as continuity equation) constitutes a better model $[3,6,15]$. This data model reads:

$$
\frac{d I}{d t}+I \operatorname{div} \mathbf{w}=0
$$

Such a constraint relates the effect of a divergent motion to a brightness change. By this way, it is possible to modelize the effect of the apparent disappearance/appearance of matter caused by $3 \mathrm{D}$ motions which are not in the visualization plane. For a null divergence this data model reduces exactly to the usual brightness consistency equation.

For long range displacements (i.e. fast flows or long time latency between two images as in meteorology) an integrated form of this constraint can be obtained[6]:

$$
I(\mathbf{x}+\mathbf{w}(\mathbf{x}), t+1) \exp (\operatorname{div} \mathbf{w}(\mathbf{x}))-I(\mathbf{x}, t)=0 .
$$

According to this constraint the displaced image at time $t+$ 1 is related to the image at time $t$ by a scale factor which depends on the motion divergence. This constraint comes to the standard displaced frame formulation of brightness consistency for a null divergence. Assuming this constraint holds almost everywhere on the whole image plane leads to seek a motion field minimizing the following cost function:

$\mathcal{F}(I, \mathbf{w})=\int_{\Omega}[I(\mathbf{x}+\mathbf{w}(\mathbf{x}), t+1) \exp (\operatorname{divw}(\mathbf{x}))-I(\mathbf{x}, t)]^{2} d \mathbf{x}$.

Considering such a cost function for an unknown motion field approximated through vortex and source particles representations comes down to solve the following minimization problem:

$$
\hat{\beta}=\arg \min _{\beta} \mathcal{F}(I, \mathbf{w}(\beta)),
$$

with $\beta=\left(\left\{\mathbf{x}_{i}^{\text {sol }}, \gamma_{i}^{s o l}, \epsilon_{i}^{\text {sol }}\right\}_{i=1: p},\left\{\mathbf{x}_{i}^{i r r}, \gamma_{i}^{i r r}, \epsilon_{i}^{i r r}\right\}_{i=1: q}\right)$. One seeks therefore the minimizer of the cost function $\mathcal{F}$ in terms of particles location, strength coefficients and influence domains. Due to the peculiar form of the data model this minimization problem is highly non linear. To face this difficult optimization problem we have chosen to rely on a two steps estimation process. First of all, the strength coefficients and the influence domain of each particle are estimated through a multi-resolution non linear least square minimization solved with a generalized conjugated gradient known as Fletcher-Reeves method. The particles locations are then moved according to a mean shift procedure [5]. This procedure allows us to move the set of vortex and source particles toward the modes of a probability distribution related to localized image reconstruction error. Interested readers can refer to [7] where this two alternates optimization is fully described. 
In order to introduce a time consistency of the velocity computed along the sequence we propose in the following a stochastic filtering method for the tracking of the solenoidal component of a fluid motion field.

\section{Filtering problem}

Before describing in detail the tracking technique we propose, we first recall the principles of discrete stochastic filtering. Stochastic filtering aims at estimating the inference of a sequence of random vector variables describing a target of interest at different discrete time instants. This sequence is assumed to be a discrete hidden Markov chain noted $\mathbf{x}_{0: n}=\left\{\mathbf{x}_{0}, \ldots, \mathbf{x}_{n}\right\}$, with initial distribution $p\left(\mathbf{x}_{0}\right)$ and transition distribution $p\left(\mathbf{x}_{k} \mid \mathbf{x}_{k-1}\right)$. At a current instant, the inference is done on the basis of the previous estimates and on a sequence of incomplete and noisy past measurements of the state. These measurements are here denoted $\mathbf{z}_{1: n}=\left\{\mathbf{z}_{1}, \ldots, \mathbf{z}_{n}\right\}$ and are assumed to be conditionally independent given the state sequence, with probability distribution $p\left(\mathbf{z}_{k} \mid \mathbf{x}_{k}\right)$.

Stochastic filters constitute procedures to estimate the posterior pdf $p\left(\mathbf{x}_{k} \mid \mathbf{z}_{1: k}\right)$ of the state, given all the measurements until $k$. The inference may be obtained in two stages:

- Given $p\left(\mathbf{x}_{k-1} \mid \mathbf{z}_{1: k-1}\right)$, the prediction step uses the transition distribution $p\left(\mathbf{x}_{k} \mid \mathbf{x}_{k-1}\right)$ to make a first approximation of the next state:

$$
p\left(\mathbf{x}_{k} \mid \mathbf{z}_{1: k-1}\right)=\int p\left(\mathbf{x}_{k} \mid \mathbf{x}_{k-1}\right) p\left(\mathbf{x}_{k-1} \mid \mathbf{z}_{1: k-1}\right) d \mathbf{x}_{k-1}
$$

- The likelihood $p\left(\mathbf{z}_{k} \mid \mathbf{x}_{k}\right)$ of a new observation $\mathbf{z}_{k}$ is used to update the posterior pdf at instant $k$ :

$$
p\left(\mathbf{x}_{k} \mid \mathbf{z}_{1: k}\right)=\frac{p\left(\mathbf{z}_{k} \mid \mathbf{x}_{k}\right) p\left(\mathbf{x}_{k} \mid \mathbf{z}_{1: k-1}\right)}{\int p\left(\mathbf{z}_{k} \mid \mathbf{x}_{k}\right) p\left(\mathbf{x}_{k} \mid \mathbf{z}_{1: k-1}\right) d \mathbf{x}_{k}}
$$

In the case of a linear Gaussian model where the posterior distribution can be analytically computed, the filtering problem is solved with the help of the well-known KalmanBucy filter. In a non linear/non Gaussian case, particle filtering authorizes a recursive Monte-Carlo approximation of the posterior density. Particle filters approximate the posterior density $p\left(\mathbf{x}_{0: k} \mid \mathbf{z}_{1: k}\right)$ by a weighted sum centered on elements of state space called particles $\left\{\mathbf{x}_{0: k}^{(i)}\right\}$. These particles are hypothetic trajectories of the state with initial condition $\mathbf{x}_{0}$, they have nothing to do with the source/vortex particles we introduced here. At each iteration $k$, the set of particles is drawn from an approximation of the unknown posterior distribution called the importance function and denoted $\pi\left(\mathbf{x}_{0: k} \mid \mathbf{z}_{1: k}\right)$. A weight is then computed for each particle, from the measurement likelihood. Assuming the importance function can be factorized as follows: $\pi\left(\mathbf{x}_{0: k} \mid \mathbf{z}_{1: k}\right)=\pi\left(\mathbf{x}_{0: k-1} \mid \mathbf{z}_{1: k-1}\right) \pi\left(\mathbf{x}_{k} \mid \mathbf{x}_{0: k-1}, \mathbf{z}_{1: k}\right)$, the method becomes sequential, and is usually denoted as $s e$ quential importance sampling filter $[9,11]$. During the prediction step, $\mathbf{x}_{k}^{(i)}$ is sampled from $\pi\left(\mathbf{x}_{k} \mid \mathbf{x}_{0: k-1}, \mathbf{z}_{1: k}\right)$, and the trajectory $\left\{\mathbf{x}_{0: k-1}^{(i)}\right\}$ is augmented with $\mathbf{x}_{k}^{(i)}$. The update step consists then in a recursive evaluation of each weight:

$$
w_{k}^{(i)}=w_{k}^{(i-1)} \frac{p\left(\mathbf{z}_{k} \mid \mathbf{x}_{k}^{(i)}\right) p\left(\mathbf{x}_{k}^{(i)} \mid \mathbf{x}_{k-1}^{(i)}\right)}{\pi\left(\mathbf{x}_{k}^{(i)} \mid \mathbf{x}_{0: k-1}^{(i)}, \mathbf{z}_{1: k}\right)} .
$$

The Monte-Carlo estimate of the posterior pdf $p\left(\mathbf{x}_{k} \mid \mathbf{z}_{1: k}\right)$ is then:

$$
\hat{p}\left(\mathbf{x}_{k} \mid \mathbf{z}_{1: k}\right)=\sum_{i=1}^{N} w_{k}^{(i)} \delta\left(\mathbf{x}_{k}-\mathbf{x}_{k}^{(i)}\right) .
$$

In practice, it is well known that the particle filtering method leads to an increase over time of the weights variance and to a decrease of the number of significant particles. A solution to tackle this problem can be to minimize the variance of the weights by introducing the so-called optimal importance function $p\left(\mathbf{x}_{k} \mid \mathbf{x}_{k-1}^{(i)}, \mathbf{z}_{k}\right)[1,9]$. This optimal function is unfortunately only available for a linear measure equation with Gaussian or mixture of Gaussian likelihood [1]. When such optimal importance function is not available, the importance function is often simply set to the prediction density: $\pi\left(\mathbf{x}_{k} \mid \mathbf{x}_{0: k-1}^{(i)}, \mathbf{z}_{1: k}\right)=p\left(\mathbf{x}_{k} \mid \mathbf{x}_{k-1}^{(i)}\right)$. In that case, the recursive formulation of the weights $w_{k}^{(i)}$ simplifies as:

$$
w_{k}^{(i)}=w_{k}^{(i-1)} p\left(\mathbf{z}_{k} \mid \mathbf{x}_{k}^{(i)}\right) .
$$

Another procedure used to avoid degeneracy consists in resampling the particles. Resampling procedures consist in replacing trajectories with small normalized weights with those associated to stronger weights.

\section{Application to vortex structures tracking}

In this section we show how such a scheme can be adapted to track the solenoidal component (ie. the vortex particles) of the motion field. For sake of simplicity we assume here that the whole motion field is divergence free and therefore reduces to its solenoidal component.

\subsection{Dynamic of the vortex particles}

Let us recall that fluid flows dynamic can be described by the Navier-Stokes equation in its velocity-vorticity formulation:

$$
\frac{\partial \xi}{\partial t}+(\mathbf{w} \cdot \nabla) \xi=\nu \triangle \xi
$$


where $\mathbf{w}=\mathbf{w}(\mathbf{x}, t)$ is the velocity and $\xi$ denotes the vorticity, defined as $\xi=$ curl w. $\nu$ is the viscosity coefficient of the fluid. At each time step, the vorticity variation is described by an advection-diffusion equation. This equation may be solved by two distinct steps. This way to handle this equation, - known as viscous splitting method [4] -, enables to treat successively the non-viscous and the viscous part of the equation. The first step is the convection part and corresponds to the left part of (12). The second is the diffusion part and is related to the right part.

If $\mathbf{x}_{i}$ denote the center of a vortex particle as defined in section (2.2), the convection and diffusion parts respectively described read:

$$
\frac{d \mathbf{x}_{i}}{d t}=\mathbf{w}\left(\mathbf{x}_{i}\right) \quad \text { and } \quad \frac{d \xi\left(\mathbf{x}_{i}\right)}{d t}=0
$$

and

$$
\frac{d \mathbf{x}_{i}}{d t}=0 \quad \text { and } \quad \frac{d \xi\left(\mathbf{x}_{i}\right)}{d t}=\nu \triangle \xi\left(\mathbf{x}_{i}\right)
$$

The total transport of vorticity due to convection and diffusion is finally obtained by combination of these two solutions. The convection step is solved through a forward integration. For fixed strength and fixed influence domain the successive locations of the particles and the underlying velocity field described is obtained from equation (7) in section (2.2). The diffusion part can be solved efficiently by Chorin's random walk method [4]. This method relies on the probabilistic interpretation of the solution of the diffusion equation $\frac{d \xi\left(\mathbf{x}_{i}\right)}{d t}=\nu \triangle \xi\left(\mathbf{x}_{i}\right)$ and on the relation between $\mathrm{d}$ iffusion and Brownian motion. In fact, it can be shown that a large set of particles undergoing Brownian motion provides a good approximation to the heat equation [13]. The principle of Chorin's random walk method associated to a viscous splitting of the vorticity advection-diffusion equation consists then in transporting forward the vortex particles by their own induced velocity field (convection step) and to add an appropriate Brownian perturbation to simulate the diffusion.

Let $\mathbf{X}_{t}$ denote the $\mathbb{R}^{2 p}$-vector gathering all the vortex particles locations $\left\{\mathbf{x}_{i}\right\}_{i=1: p}$ at time $t$. Let $\mathbf{w}\left(\mathbf{X}_{t}\right)$ represent the vector of the velocities evaluated at these locations. The stochastic process $\mathbf{X}=\left(\mathbf{X}_{t}\right)_{t \geq 0}$ evolves then according to the following Itô diffusion process:

$$
d \mathbf{X}_{t}=\mathbf{w}\left(\mathbf{X}_{t}\right) d t+\sigma d B_{t}
$$

where $d B$ is a $2 p$-dimensional Brownian motion with independent components, and $\sigma=\sqrt{2 \nu}+\eta$, where $\eta$ express the uncertainty on the model. Discretizing such a stochastic differential equation with an Euler scheme and initial condition $X_{0}^{k}=X^{k}$ gives:

$$
\mathbf{X}_{j}^{k}=\mathbf{X}_{j-\Delta t}^{k}+\mathbf{w}\left(\mathbf{X}_{j-\Delta t}\right) \Delta t+\mathbf{v}_{j},
$$

where $\mathbf{v}_{j}$ are zero mean independent Gaussian noises, with standard deviation $\sigma$. The evolution of the state $\mathbf{X}$ between two frame instants $k$ and $k+1$ and for discretization steps $\Delta t$ is represented by the following transition equation:

$$
p\left(\mathbf{X}_{j}^{k} \mid \mathbf{X}_{j-\Delta t}^{k}\right)=\mathcal{N}\left(\mathbf{X}_{j-\Delta t}^{k}+\mathbf{w}\left(\mathbf{X}_{j-\Delta t}^{k}\right) \Delta t, \sigma \Delta t \mathbb{I}_{2 p}\right),
$$

where $\mathbb{I}_{2 p}$ denotes the $2 p \times 2 p$ identity matrix.

\subsection{Measurement equation}

The set $\mathbf{z}_{1: n}=\left\{\mathbf{z}_{1}, \ldots, \mathbf{z}_{n}\right\}$ of measurements is extracted from the image sequence. At each step $k$, the measuremen$\mathrm{t}$ equation is constructed from the estimated field $\mathrm{w}^{k}$ (obtained from the predicted positions of the vortex particles) and the pair of images $\left(I_{k}, I_{k+1}\right)$. We index $\mathbf{w}$ by $k$ to emphasize the fact that it depends on the positions of all the vortex particles at time $k$.

In order to devise a measurement equation likelihood, a region $R_{i}$ is defined around each vortex particle $\mathbf{x}_{i}$. This region is in practice fixed to the influence domain of the corresponding particle. Assuming then a brightness consistency up to a Gaussian noise for all points belonging to region $R_{i}$, we have:

$$
I_{k}(\mathbf{x})=I_{k+1}\left(\mathbf{x}+\mathbf{w}^{k}(\mathbf{x})\right)+u_{k},
$$

where $u_{k}$ is a zero mean Gaussian noise of variance $\sigma^{2}$.

Noting $R$ as the union of all the regions $R_{i}$ and $\mathbf{z}_{k}$ as the vector gathering all the luminance values $I_{k}(\mathbf{x})$ for all $\mathbf{x}$ in $R$ and assuming that $I_{k}(\mathbf{x})$ and $I_{k}\left(\mathbf{x}^{\prime}\right)$ are independent conditionally to $\mathbf{X}_{k} \forall\left(\mathbf{x}, \mathbf{x}^{\prime}\right) \in R$, the likelihood reads:

$$
p\left(\mathbf{z}_{k} \mid \mathbf{X}_{k}\right)=\prod_{\mathbf{x} \in R} p\left(I_{k}(\mathbf{x}) \mid \mathbf{X}_{k}\right)
$$

and consequently

$$
p\left(\mathbf{z}_{k} \mid \mathbf{X}_{k}\right) \propto \exp \left(-\int_{R} \frac{\left(I_{k}(\mathbf{x})-I_{k+1}\left(\mathbf{x}+\mathbf{w}^{k}(\mathbf{x})\right)^{2}\right.}{2 \sigma^{2}} d \mathbf{x}\right) .
$$

\subsection{Tracking by non linear filtering}

The filtering method presented in section 3 is used to track the set $\left\{\mathbf{x}_{i}\right\}_{i=1: p}$ of vortex particles. The highly non linear nature of the state dynamic and of the likelihood requires the use of the particle filtering method. The optimal importance function is obviously not available in our case, we thus set the importance function to the prediction density and use formula (11) to evaluate the particles weights.

The prediction and update steps of the particle filter algorithm are performed iteratively, together with a resampling of the particles cloud when necessary. 
At $k=0$, the initial distribution $p\left(\mathbf{X}_{0}\right)$ of the set of vortex particles is given by the estimation method presented in section 2.3. In fact, the method gives a set of positions for the vortex particles and the amount of vorticity carried by each particle (through the estimated strength coefficient and influence parameter). This initial distribution gives the initial representation of the solenoidal component.

Knowing $p\left(\mathbf{X}_{k-1} \mid \mathbf{z}_{k-1}\right)$, the filtering particles are sampled from (17) with $X_{j=0}^{k-1}=X^{k-1}$ as initial condition. The sampling is done iteratively until $j=N$, (with $N \Delta t=1$ ). It is important to outline that no update step can be performed between two frame instants as no observation is available within $j=0$ and $j=N$. At $j=N$ a new observation becomes available and the particles weights are updated according to equation (11), with $p\left(\mathbf{z}_{k} \mid \mathbf{X}_{k}^{(i)}\right)$ given by (20).

\section{Results}

In this section we show results obtained on a 60 frames real world sequence showing a vortex launch at tip of airplane wing. The tracker is initialized with the help of the estimation method described in section (2.3). Such a method provides us an initial set of locations for the vortex particles, together with a description of the vorticity distribution in terms of strength and amplitude parameters associated to each particle.

The initial state is presented in figure (1). On this figure we have plotted the initial vector field, the first image of the sequence and the corresponding vorticity map. On this latter map one can see a primary vortex and a smaller secondary vortex turning around the first vortex. This result has been obtained with a set of 15 vortex particles.

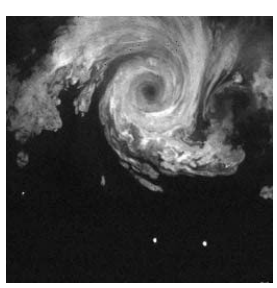

(a)

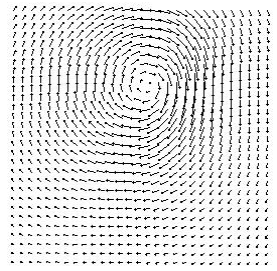

(b)

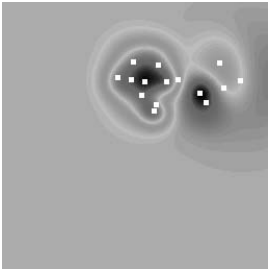

(c)
Figure 1: (a) First image of the sequence; (b) motion field estimated at time $\mathrm{k}=0$, with the method presented in section 2.3; (c) corresponding vorticity distribution, set of vortex particles used to initialize the tracking.

To demonstrate the efficiency of the tracking we compare the solution obtained only considering the dynamic with the filtered solution. Figure (2) shows the result obtained only propagating the initial set of vortex particles according to the dynamic equation described in section (15).

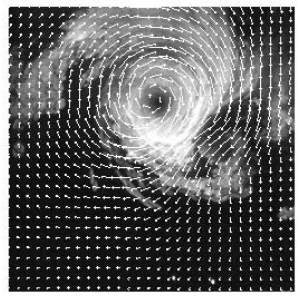

$k=10$

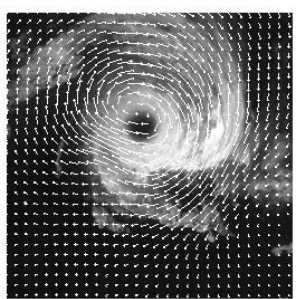

$k=30$

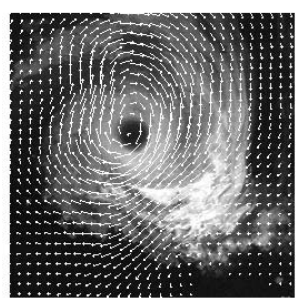

$$
k=50
$$

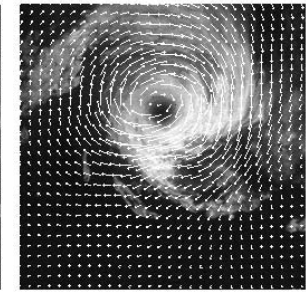

$k=20$

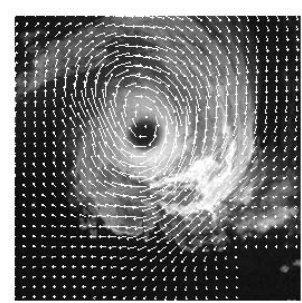

$k=40$

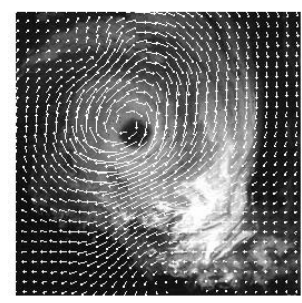

$k=60$
Figure 2: Evolution of the motion field obtained by propagating the initial vortex particles according to the Itô diffusion process (15).

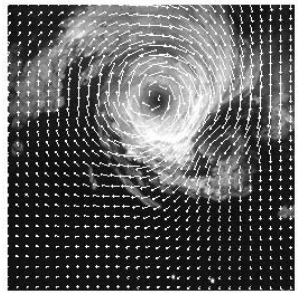

$k=10$

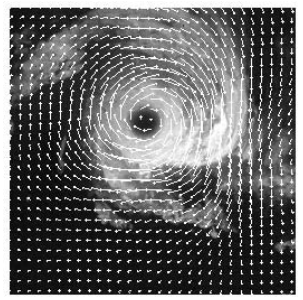

$k=30$

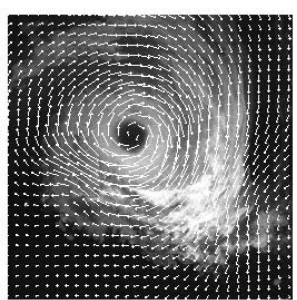

$k=50$

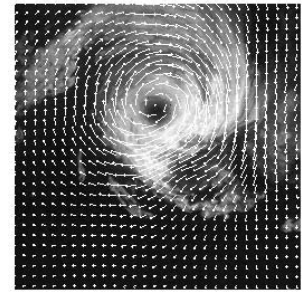

$k=20$

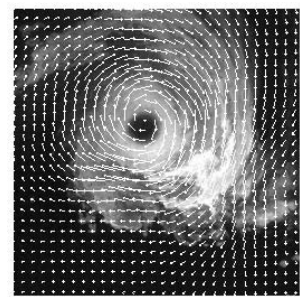

$k=40$

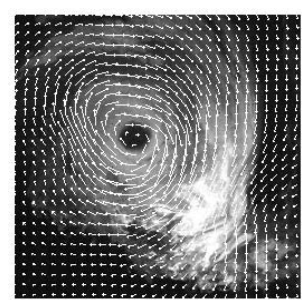

$k=60$
Figure 3: Evolution of the motion field obtained by particle filtering. 
Figure (3) shows the result obtained by the non linear filtering method described in the previous section. The filter has been run with the same initial conditions. As can be observed from fig. (2), the motion fields obtained considering only the dynamic show a significant drift of the vortex. In addition the vortex degenerates quickly and shows, from frame \#30, improper elongations which are not visible on the image sequence.

As can be seen on fig. (3), the solution recovered by the tracking method is visually much more satisfying. The vortex motion is well reconstructed at each frame instant. No vortex drift is observed anymore, as illustrates also the vorticity maps comparison on fig. (4). The deformation of the vortex is also well reconstructed and fit well the photometric vortex contours.
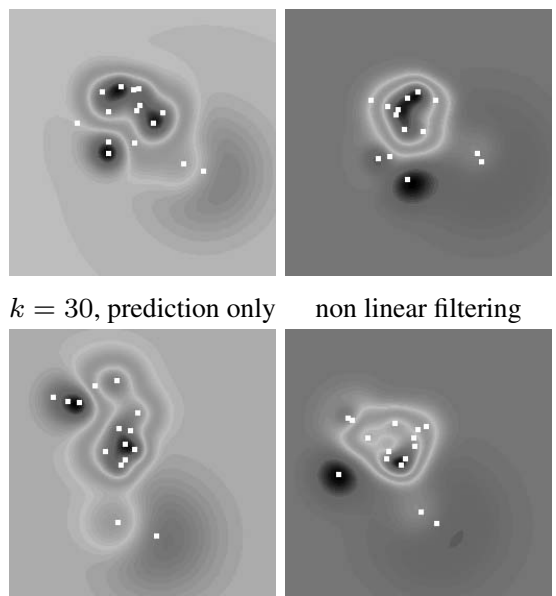

non linear filtering

$k=50$, prediction only

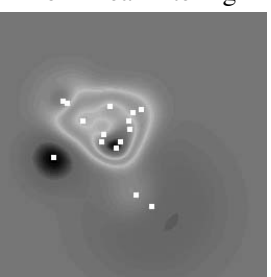

non linear filtering

Figure 4: Comparison of the vorticity maps for two different times of the tracking.

\section{Conclusion}

In this paper we have proposed a non linear stochastic filter for the tracking of fluid motion. The tracking is based on a low dimensional representation of the velocity field obtained through a discretization of the vorticity and divergence maps. To the best of our knowledge, it is the first time that a method to track motion field features is proposed. Beyond the tracking, such a method allows to recover a set of consistent velocity fields for a whole sequence. This method is nevertheless restricted to solenoidal fields. When diverging motions are present the corresponding irrotational component must be either estimated at each frame instant or tracked with another dynamic expression.

\section{Acknowledgement}

This work was supported by the European community through the IST Fet open FLUID project (http://fluid.irisa.fr).

\section{References}

[1] E. Arnaud and E. Mémin. Optimal importance sampling for tracking in image sequences: application to point tracking. In Proc. Europ. Conf. Comp. Vision, May 2004.

[2] J. Barron, D. Fleet, and S. Beauchemin. Performance of optical flow techniques. Int. J. Comp. Vision, 12(1):43-77, 1994.

[3] D. Béréziat, I. Herlin, and L. Younes. A generalized optical flow constraint and its physical interpretation. In Proc. Conf. Comp. Vision Pattern Rec., volume 2, pages 487-492, Hilton Head Island, South Carolina, USA, 2000.

[4] A. Chorin. Numerical study of slightly viscous flow. J. Fluid Mech., 57:785-796, 1973.

[5] D. Comaniciu and P. Meer. Mean shift: A robust approach toward feature space analysis. IEEE Trans. Pattern Analysis Machine Intelligence, 24(5):603-619, 2002.

[6] T. Corpetti, E. Mémin, and P. Pérez. Dense estimation of fluid flows. IEEE Trans. Pattern Anal. Machine Intell., 24(3):365380, 2002.

[7] A. Cuzol and E. Mémin. Vortex and source particles for fluid motion estimation. In Scale Space '05, LNCS 3459, pages 254-266, 2005.

[8] P. Del Moral, J. Jacod, Ph. Protter. The monte-carlo method for filtering with discrete-time observations. Probab. Theory Relat. Fields, 120:346-368, 2001.

[9] A. Doucet, S. Godsill, and C. Andrieu. On sequential monte carlo sampling methods for bayesian filtering. Statistics and Computing, 10(3):197-208, 2000.

[10] G. Fanerback. Very high accuracy velocity estimation using orientation tensors, parametric motion, and segmentation of the motion field. In Proc. Int. Conf. Computer Vision, pages $5-26,1999$.

[11] M. Isard and A. Blake. Condensation - conditional density propagation for visual tracking. Int. J. Computer Vision, 29(1):5-28, 1998.

[12] A. Leonard. Vortex methods for flow simulation. J. Comp. Phys., 37, 1980.

[13] B. Oksendal. Stochastic differential equations. SpringerVerlag, 1998.

[14] J. Vermaak P. Pérez and A. Blake. Data fusion for visual tracking. Proc. IEEE, 92(3):495-513, 2004.

[15] B.G. Schunk. The motion constraint equation for optical flow. In Proc. Int. Conf. Pattern Recognition, pages 20-22, Montreal, 1984.

[16] J. Weickert and C. Schnörr. Variational optic-flow computation with a spatio-temporal smoothness constraint. J. Mathematical Imaging and Vision, 14(3):245-255, 2001. 